\title{
THE DISCREPANCY OF SOME REAL SEQUENCES
}

\author{
H. KAMARUL HAILI and R. NAIR
}

\section{Abstract}

Let $\left(\lambda_{n}\right)_{n \geq 0}$ be a sequence of real numbers such that there exists $\delta>0$ such that $\left|\lambda_{n+1}-\lambda_{n}\right| \geq \delta$, $n=0,1, \ldots$. For a real number $y$ let $\{y\}$ denote its fractional part. Also, for the real number $x$ let $D(N, x)$ denote the discrepancy of the numbers $\left\{\lambda_{0} x\right\}, \ldots,\left\{\lambda_{N-1} x\right\}$. We show that given $\varepsilon>0$,

$$
D(N, x)=o\left(N^{-\frac{1}{2}}(\log N)^{\frac{3}{2}+\varepsilon}\right)
$$

almost everywhere with respect to Lebesgue measure.

\section{Introduction}

Recall that a sequence of real numbers $\left(x_{n}\right)_{n=0}^{\infty}$ is uniformly distributed modulo one if for each interval $I$ contained in $[0,1)$, that is closed on the left and open on the right,

$$
\lim _{N \rightarrow \infty} \frac{1}{N} \sum_{n=0}^{N-1} \chi_{I}\left(\left\{x_{n}\right\}\right)=|I| .
$$

Here $\chi_{I}$ denotes the characteristic function of the interval $I$ and $|I|$ its length. Also $\{y\}$ is the fractional part of a real number $y$. For a finite set of real numbers $x_{0}, \ldots, x_{N-1}$, their discrepancy is

$$
D\left(x_{0}, \ldots, x_{N-1}\right)=\sup _{I \subseteq[0,1)}\left|\frac{1}{N} \sum_{n=0}^{N-1} \chi_{I}\left(\left\{x_{n}\right\}\right)-\right| I|| \quad(N=1,2, \ldots)
$$

where the supremum is taken over all intervals $I$, closed on the left and open on the right. The discrepancy of the numbers $x_{0}, \ldots, x_{N-1}$ tends to zero as $N$ tends to infinity if and only if the sequence $\left(x_{n}\right)_{n=0}^{\infty}$ is uniformly distributed modulo one. This means that for a uniformly distributed sequence of real numbers $\left(x_{n}\right)_{n=0}^{\infty}$, as $N$ tends to infinity, the rate for decay of $D\left(x_{0}, \ldots, x_{N-1}\right)$ provides a measure of the degree of uniformity of distribution. As usual if a property holds except for a set of Lebesgue measure zero, it is said to hold almost everywhere, abbreviated 'a.e.'. Throughout the paper $C$ will denote a

Received September 11, 1997; in revised form February 25, 2002. 
positive constant which will not necessarily be the same at each occurence. Let $\left(\lambda_{n}\right)_{n=0}^{\infty}$ denote a sequence of real numbers such that there exists $\delta>0$ such that

$$
\left|\lambda_{n+1}-\lambda_{n}\right| \geq \delta>0 \quad(n=0,1, \ldots) .
$$

In this paper we prove two theorems.

THeOREM 1.1. Let

$$
D(N, x)=D\left(\left\{\lambda_{0} x\right\}, \ldots,\left\{\lambda_{N-1} x\right\}\right) \quad(N=0,1, \ldots) .
$$

Then given $\varepsilon>0$,

$$
D(N, x)=o\left(N^{-\frac{1}{2}}(\log N)^{\frac{3}{2}+\varepsilon}\right) \text { a.e. }
$$

One might wish to consider sets other than intervals. However a famous example of J. M. Marstrand [5] states that there exist subsets $B$ and $A(B)$ of $[0,1)$, both of positive Lebesgue measure, such that if $x$ is in $A(B)$, then the averages

$$
\frac{1}{N} \sum_{j=0}^{N-1} \chi_{B}(\{j x\}) \quad(N=1,2, \ldots),
$$

do not converge to the Lebesgue measure of $B$. With suitable restrictions on $B$ however positive results are however possible.

THEOREM 1.2. Let $\left(R_{k}\right)_{k=1}^{\infty}$ be a collection of disjoint subintervals of $[0,1)$ such that

$$
\left|R_{k}\right|=O\left(a^{-k}\right)
$$

for some $a>1$, and let

$$
B=\bigcup_{k=1}^{\infty} R_{k} .
$$

Then given $\varepsilon>0$, there exists $N_{0}=N_{0}(x, \varepsilon)$ such that if $N>N_{0}$

$$
\left|\frac{1}{N} \sum_{n=0}^{N-1} \chi_{B}\left(\left\{\lambda_{n} x\right\}\right)-\right| B||<N^{-\frac{1}{2}}(\log N)^{\frac{5}{2}+\varepsilon} \text { a.e. }
$$

Theorem 1.1, but with $\frac{5}{2}$ instead $\frac{3}{2}$ in the power of $\log N$ appears in [2] and in [1] but with the restriction that $\left(\lambda_{n}\right)_{n=0}^{\infty}$ are integers. The extension of these results to Theorem 1.1 is made possible by Lemma 2.2 below, which is a consequence of the maximal inequality for the Carleson-Hunt inequality and the 
properties of Vaaler polynomials [6]. The methods used to prove Theorem 1.1 are the basis of the proof of Theorem 1.2.

ADDED IN PROOF. Theorem 1.1 also appears in G. Harmans book "Metric Number Theory”, London Math. Soc. Monographs (N.S.) 18, 1988.

\section{Proof of Theorem 1.1}

To prove Theorem 1.1, we need the following lemmas.

Lemma 2.1 ([3]). Given real numbers $x_{0}, \ldots, x_{N-1}$, there exists $C>0$ such that for all natural numbers $L$

$$
N D\left(x_{0}, \ldots, x_{N-1}\right) \leq C\left(\frac{N}{L}+\sum_{h=1}^{L} \frac{1}{h}\left|\frac{1}{N} \sum_{n=0}^{N-1} e^{2 \pi i h x_{n}}\right|\right) .
$$

Lemma 2.2 ([6]). Suppose we are given $\delta>0$, real numbers $\left(\lambda_{n}\right)_{n=0}^{N-1}$ such that $\lambda_{n+1}-\lambda_{n} \geq \delta>0$, real numbers $T$ and $T_{0}$ with $T>0$ and complex numbers $\left(a_{n}\right)_{n=1}^{N}$. Then there exists $C>0$ such that

$$
\int_{T_{0}}^{T_{0}+T}\left\{\max _{0 \leq v \leq N-1}\left|\sum_{n=0}^{v} a_{n} e^{i \lambda_{n} t}\right|^{2}\right\} d t \leq C\left(T+2 \pi \delta^{-1}\right) \sum_{n=0}^{N-1}\left|a_{n}\right|^{2} .
$$

Note that Lemma 2.2 in the special case where the $\left(\lambda_{n}\right)_{n=1}^{\infty}$ are all integers reduces to the maximal inequality of Carleson-Hunt [4]. Plainly in proving Theorem 1.1, we may without loss of generality assume $x$ belongs a finite interval $\left[T_{0}, T_{0}+T\right]$. Let $f:\left[T_{0}, T_{0}+T\right] \rightarrow \mathrm{R}$ be square integrable and let

$$
\|f\|=\left(\frac{1}{T} \int_{T_{0}}^{T_{0}+T}|f|^{2} d x\right)^{\frac{1}{2}} .
$$

Then by applying Minkowski’s inequality to Lemma 2.1,

$$
\left\|\max _{1 \leq v \leq N} v D(v, x)\right\| \leq C\left(\frac{N}{L}+\sum_{h=1}^{L} \frac{1}{h}\left\|\max _{1 \leq v \leq N}\left|\sum_{n=1}^{v} e^{2 \pi i \lambda_{n} x}\right|\right\|\right),
$$

whence by Lemma 2.2

$$
\left\|\max _{1 \leq v \leq N} v D(v, x)\right\| \leq C\left(\frac{N}{L}+\sum_{h=1}^{L} \frac{1}{h} N^{\frac{1}{2}}\right) .
$$


Choosing $L$ optimally this gives

$$
\left\|\max _{1 \leq v \leq N} v D(v, x)\right\| \leq C N^{\frac{1}{2}}(\log N) .
$$

To deduce Theorem 1.1, we argue as in [2] and let

$$
E(\varepsilon)=\left\{x \in\left[T_{0}, T_{0}+T\right]: \limsup _{l \rightarrow \infty} \frac{l D(l, x)}{f(l, \varepsilon)}>0\right\},
$$

where

$$
f(l, \varepsilon)=l^{\frac{1}{2}}(\log N)^{\frac{3}{2}+\varepsilon} .
$$

We need to show that the Lebesgue measure $|E(\varepsilon)|$ of $E(\varepsilon)$ is zero for all $\varepsilon>0$. Set

$$
A_{s}(\varepsilon)=\left\{x \in\left[T_{0}, T_{0}+T\right]: \max _{1 \leq l \leq 4^{s}} l D(l, x)>\frac{1}{4} f\left(4^{s}, \frac{\varepsilon}{2}\right)\right\} .
$$

If $x \in E(\varepsilon)$ then there exist $c(\varepsilon, x)>0$ and arbitrarily large positive integers $s$ such that for some integer $l$ in $\left[4^{s-1}, 4^{s}\right)$

$$
l D(l, x) \geq c(\varepsilon, x) f\left(4^{s-1}, \varepsilon\right) \geq f\left(4^{s-1}, \frac{\varepsilon}{2}\right) \geq \frac{1}{4} f\left(4^{s}, \frac{\varepsilon}{2}\right),
$$

The last inequality here being evident from the identity

$$
f\left(4^{s-1}, \frac{\varepsilon}{2}\right)=\frac{1}{2}\left(\frac{\log s-1}{\log s}\right)^{\frac{3}{2}+\frac{\varepsilon}{2}} f\left(4^{s}, \frac{\varepsilon}{2}\right)
$$

and the fact that for large enough $s$ we have $\frac{1}{2}\left(\frac{\log s-1}{\log s}\right)^{\frac{3}{2}+\frac{\varepsilon}{2}}>\frac{1}{4}$. In particular, we know that for infinitely many $s$,

$$
\max _{1 \leq l \leq 4^{s}}|l D(l, x)|>\frac{1}{4} f\left(4^{s-1}, \frac{\varepsilon}{2}\right) .
$$

This tells us that $x$ is in infinitely many of the sets $A_{s}(\varepsilon)$. Hence we can conclude that

$$
E(\varepsilon) \subseteq \bigcap_{r=1}^{\infty} \bigcup_{s=r}^{\infty} A_{s}(\varepsilon)
$$

From (2.1) there exists $C>0$ such that

$$
\left|A_{s}(\varepsilon)\right|\left(f\left(4^{s}, \frac{\varepsilon}{2}\right)\right)^{2} \leq C 4^{s}\left(\log 4^{s}\right)^{2} .
$$


Hence

$$
\left|A_{s}(\varepsilon)\right| \leq \frac{C}{S^{1+\varepsilon}}
$$

and

$$
\sum_{s=1}^{\infty}\left|A_{s}(\varepsilon)\right|<\infty
$$

so by the Borel-Cantelli Lemma, Theorem 1.1 is proved.

\section{Proof of Theorem 1.2}

For

$$
z(N)=\log _{a} N \quad(N=1,2, \ldots)
$$

let

$$
t(N)=\bigcup_{1 \leq k \leq z(N)} R_{k} \quad(N=1,2, \ldots)
$$

and

$$
s(N)=\bigcup_{k>z(N)} R_{k} \quad(N=1,2, \ldots) .
$$

Note that if, for any $S \subseteq[0,1)$ we set

$$
K(S, l, x)=\frac{1}{l} \sum_{n=0}^{l-1} \chi_{S}\left(\left\{\lambda_{n} x\right\}\right)-|S| \quad(l=1,2, \ldots),
$$

then

$$
K(B, l, x)=K(t(N), l, x)+K(s(N), l, x) .
$$

Hence

$$
\begin{aligned}
& \left\|\max _{0 \leq l \leq N-1} K(B, l, x)\right\| \\
& \quad \leq\left\|\max _{0 \leq l \leq N-1}|K(t(N), l, x)|\right\|+\left\|\max _{0 \leq l \leq N-1}|K(s(N), l, x)|\right\| .
\end{aligned}
$$

Note that

$$
K(t(N), l, x)=\sum_{1 \leq k \leq z(N)}\left(\sum_{j=0}^{l-1} \chi_{R_{k}}\left(\left\{\lambda_{j} x\right\}\right)-l\left|R_{k}\right|\right)
$$

so,

$$
\left\|\max _{0 \leq l \leq N-1} K(t(N), l, x)\right\| \leq z(N)\left\|\max _{0 \leq l \leq N-1} l D(l, x)\right\| .
$$


This by (2.1) gives

$$
\left\|\max _{0 \leq l \leq N-1} K(t(N), l, x)\right\| \leq C z(N) N^{\frac{1}{2}}(\log N) .
$$

By definition

$$
\left\|\max _{0 \leq l \leq N-1}|K(s(N), l, x)|\right\|=\left\|\max _{0 \leq l \leq N-1}\left|\sum_{j=0}^{l-1} \chi_{s(N)}\left(\left\{\lambda_{j} x\right\}\right)-l\right| s(N)||\right\| .
$$

Also evidently

$$
\left\|\max _{0 \leq l \leq N-1}\left|\sum_{j=0}^{l-1} \chi_{s(N)}\left(\left\{\lambda_{j} x\right\}\right)-l\right| s(N)||\right\| \leq \sum_{j=0}^{N-1}\left\|\chi_{s(N)}\left(\left\{\lambda_{j} x\right\}\right)\right\|+N|s(N)| .
$$

As $\chi_{R_{k}}^{2}=\chi_{R_{k}}$, if

$$
E_{k, j}=\left\{x \in\left[T_{0}, T_{0}+T\right]:\left\{\lambda_{j} x\right\} \in R_{k}\right\},
$$

we see that

$$
\sum_{j=0}^{N-1}\left\|\chi_{s(N)}\left(\left\{\lambda_{j} x\right\}\right)\right\| \leq C \sum_{j=0}^{N-1}\left(\sum_{k>z(N)}\left|E_{k, j}\right|\right)^{\frac{1}{2}} .
$$

It is very easy to check that there exists $C=C\left(T_{0}, T\right)>0$ such that

$$
\left|E_{k, j}\right| \leq C\left|R_{k}\right| \text {. }
$$

This means that

$$
\left\|\max _{0 \leq l \leq N-1}|K(s(N), l, x)|\right\| \leq C N\left(|s(N)|^{\frac{1}{2}}+|s(N)|\right) .
$$

Also

$$
|s(N)|=\sum_{k>z(N)}\left|R_{k}\right| \leq C \sum_{k>z(N)} a^{-k} \leq C a^{-z(N)} .
$$

So we have shown that

$$
\left\|\max _{0 \leq l \leq N-1}|K(B, l, x)|\right\| \leq C N^{\frac{1}{2}}(\log N)^{2} .
$$

The argument of the previous section now gives Theorem 1.2. 


\section{REFERENCES}

1. Baker, R. C., Metric number theory and the large sieve, J. London Math. Soc (2) 24 (1981), $34-40$.

2. Cassels, J. W. S., Some metrical theorems in diophantine approximation III, Proc. Cambridge Philos. Soc. 46 (1950), 219-225.

3. Erdős, P. and Turan, P., On a problem in the theory of uniform distribution I, II, Indag. Math. 10 (1948), 370-378, 406-413.

4. Hunt, R. A., On the convergence of Fourier series, in Orthogonal expansions and their continuous analogues, Proc. Conf. Edwardsville, Ill, 1967, 235-255, Southern Illinois University Press, Carbondale Ill (1968).

5. Marstrand, J. M.,On Khinchin's conjecture about strong uniform distribution, Proc. London Math. Soc. (3) 21 (1970), 540-556.

6. Montgomery, H. L. and Vaaler, J. D., Maximal variants obasic inequalities, Proceedings of the Congress on Number theory, (Zarautz 1984) 181-197, Univ. Pais Vasco-Euskal Herriko Unib., Bilbao, 1989.

SCHOOL OF MATHEMATICAL SCIENCES

UNIVERSITI SAINS MALAYSIA

MINDEN 11800 PENANG

MALAYSIA
MATHEMATICAL SCIENCES

UNIVERSITY OF LIVERPOOL

P.O. BOX 147

LIVERPOOL L69 7ZL

U.K. 